\title{
Ligand Dynamics in Nanocrystal Solids Studied with Quasi-Elastic Neutron Scattering
}

\author{
Maximilian Jansen, Fanni Juranyi,* Olesya Yarema, Tilo Seydel, and Vanessa Wood*
}

Cite This: ACS Nano 2021, 15, 20517-20526

Read Online

\section{ACCESS}

Џll Metrics \& More

回国 Article Recommendations

S1 Supporting Information

ABSTRACT: Nanocrystal surfaces are commonly populated by organic ligands, which play a determining role in the optical, electronic, thermal, and catalytic properties of the individual nanocrystals and their assemblies. Understanding the bonding of ligands to nanocrystal surfaces and their dynamics is therefore important for the optimization of nanocrystals for different applications. In this study, we use temperature-dependent, quasielastic neutron scattering (QENS) to investigate the dynamics of different surface bound alkanethiols in lead sulfide nanocrystal solids. We select alkanethiols with mono- and dithiol terminations, as well as different backbone types and lengths. QENS spectra are collected both on a time-offlight spectrometer and on a backscattering spectrometer, allowing us to

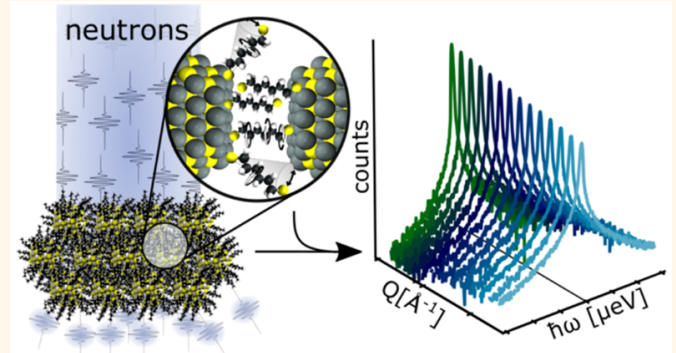
investigate ligand dynamics in a time range from a few picoseconds to nanoseconds. Through model-based analysis of the QENS data, we find that ligands can either (1) precess around a central axis, while simultaneously rotating around their own molecular axis, or (2) only undergo uniaxial rotation with no precession. We establish the percentage of ligands undergoing each type of motion, the average relaxation times, and activation energies for these motions. We determine, for example, that dithiols which link facets of neighboring nanocrystals only exhibit uniaxial rotation and that longer ligands have higher activation energies and show smaller opening angles of precession due to stronger ligand-ligand interactions. Generally, this work provides insight into the arrangement and dynamics of ligands in nanocrystal solids, which is key to understanding their mechanical and thermal properties, and, more generally, highlights the potential of QENS for studying ligand behavior.

KEYWORDS: colloidal nanocrystal, quantum dot, ligands, neutron scattering, ligand dynamics, nanoparticle assembly, QENS

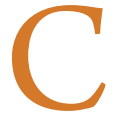
olloidal nanocrystals (NCs) are crystallites made of semiconducting or metallic materials that are $1-10$ $\mathrm{nm}$ in diameter and are surface-passivated with organic or inorganic moieties ("ligands"). Through control of the synthesis (e.g., choice of precursor, time, temperature, solvent, and ligand), the composition and size of NCs can be systematically engineered. Due to their small size, a characteristic of NCs is their large surface to volume ratio. The NC surface and its ligands thus have a significant effect on how NCs are processed (e.g., their solvation, stability in solution, and assembly from colloidal form into solids) ${ }^{1,2}$ and on their functionality (e.g., their catalytic, electronic, optical, phononic, and thermal performance). ${ }^{3-7}$ For example, for NCs used in catalysis, the surface ligands can impact the catalyst energy efficiency and selectivity. ${ }^{8-11}$ In the case of (opto)electronics, the type of ligand as well as its orientation determines the surface electrical dipole of individual NCs. This tunes the energy position of the electronic states of individual NCs as well as their assemblies and thus optical absorption and emission. ${ }^{12,13}$ Furthermore, the electronic transport properties of NC solids depend on the electronic wave function confinement of individual NCs as well as the NC packing in the NC solid (facet-to-facet alignment and spacing), all of which are determined by the ligands. ${ }^{14,15}$ Mechanical, phononic, and thermal conductivity of solids assembled from NCs are governed by the number of ligand interconnections, their orientation, and the elasticity. ${ }^{16-19}$ These examples illustrate the importance of ligands in optimizing the performance of $\mathrm{NCs}$ and $\mathrm{NC}$ thin films for different applications.

Ligands can be exchanged post-NC synthesis either when the NCs are solvated or already in a thin film solid state. Therefore, they offer a rich degree of control and selectivity with parameter choices, including the functional binding group, ligand dipole, and ligand length. ${ }^{3,20,21}$ Thus, while the ability to precisely tune NC ligand chemistry exists, judicious choice of ligands requires understanding of how they bond to

Received: October 14, 2021

Accepted: December 2, 2021

Published: December 8, 2021 

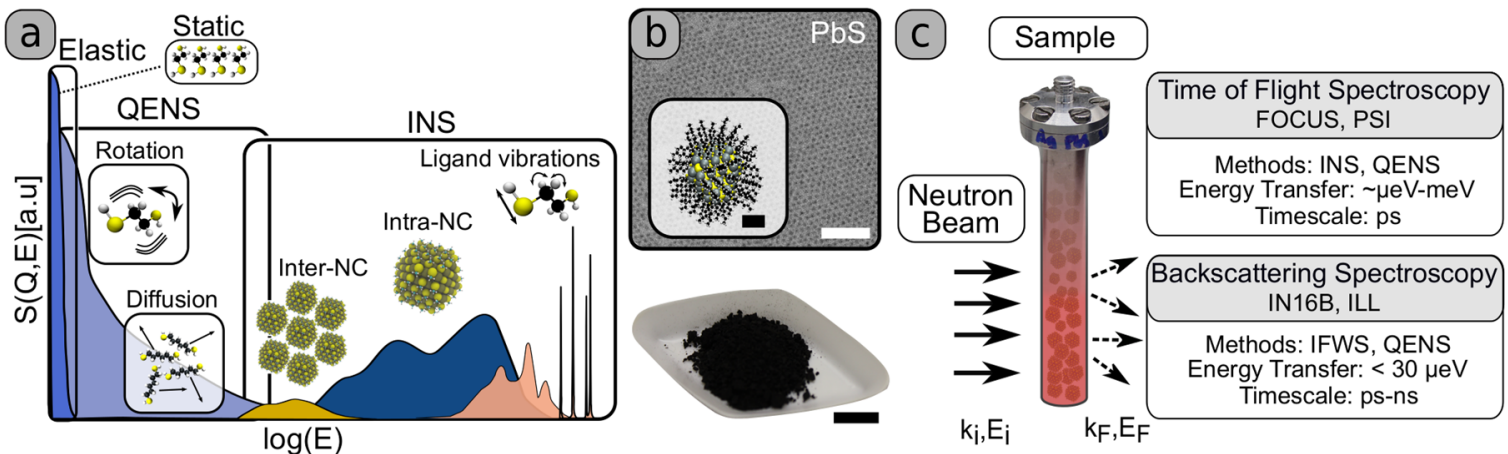

Figure 1. Neutron spectroscopy on nanocrystal (NC) solids. (a) Schematic scattering factor $S(Q E)$ intensity showing information available across the different regimes from elastic scattering, quasi-elastic neutron scattering (QENS), and inelastic neutron scattering (INS). (b) Top: TEM image of as-synthesized PbS NCs; scale bar, $50 \mathrm{~nm}$. Inset: Schematic of individual PbS NC; scale bar, $1.5 \mathrm{~nm}$. Bottom: NC powder (1.5 g) used for a neutron scattering measurement; scale bar, $5 \mathrm{~mm}$. (c) Visualization and comparison of scattering experiments and spectrometer details.

the NC surfaces and arrange to form the NC solid. Ligands and surfaces have been predominately studied by optical spectroscopy (FTIR, UV-vis), thermal analysis (DSC, TGA), surface spectroscopy (XPS), and nuclear magnetic resonance spectroscopy (NMR). ${ }^{21-26}$ Information about the effect of ligands on NC shape and interparticle spacing can be obtained indirectly by small-angle X-ray scattering (SAXS) $)^{1,27-29}$ and transmission electron microscopy (TEM). ${ }^{30}$ Because many ligands contain hydrogen, which has a large incoherent neutron scattering cross-section, they are well suited to be studied by neutron techniques. This includes small angle neutron scattering (SANS) to study the structure of the ligand shell, its solvation, and its influence on the superlattice formation. ${ }^{31-35}$ Further, neutron spectroscopy can be used to study the dynamics in NC solids: neutrons hit the sample with a well-defined wavelength, and from the statistical distribution of the momentum and energy transfer of the scattered neutrons, the dynamic structure factor, $S(Q E)$, can be obtained. The dynamic structure factor contains information about the collective (i.e., coherent scattering) and individual (i.e., incoherent scattering) motion of atoms. However, the separation of the superimposed contributions to the scattering signal from different sample components and different types of motion can pose a challenge; common contributions are illustrated in Figure 1a. Information on the crystal structure is contained in elastic scattering, for which there is no energy transfer, $S(Q E=0) .^{5}$ Inelastic neutron scattering (INS) spectra have been used to obtain information on a number of vibrational processes in NCs, ranging from interparticle vibrations ${ }^{18}$ to soft surface modes, and phonons in the nanocrystal core. ${ }^{5,35}$ Scattering centered around the elastic line at a low energy transfer of micro- to millielectronvolts is referred to as quasi-elastic neutron scattering (QENS). To date, sparsely used for studying ligands on NCs, it probes the individual, diffusive motion of atoms (in this case hydrogen) and can thus be used to develop and validate models for ligand dynamics. It is used extensively in the fields of soft matter, ${ }^{36}$ liquid crystals, ${ }^{37}$ and proteins, ${ }^{38}$ and QENS studies on NCs have examined the dynamics of surface bound ligands on $\mathrm{PbS}$ NCs in solution ${ }^{35}$ and alkanethiols on metallic clusters. ${ }^{39-41}$

Here, we perform QENS on lead sulfide ( $\mathrm{PbS}$ ) NC solids, a well-studied and widely used reference system. ${ }^{5,28,42}$ The PbS NCs grow as truncated octahedrons with ligands preferentially attaching to the Pb-rich $[111]$ facets. ${ }^{43}$ As highlighted in the TEM images in Figure $1 \mathrm{~b}$ and in the Supporting Information
(SI), the NCs have a $1.7 \pm 0.2 \mathrm{~nm}$ core radius. The NC for all samples stem from a single batch, ${ }^{44}$ so no difference in size or size distribution exists between individual samples. Together with the dominant scattering of hydrogen atoms, this allows us to attribute all observed trends to the different ligand species. We study four different ligands: 1-hexanethiol (HT), 1,6hexanedithiol (HDT), 1-dodecanethiol (DDT), and 1,4benzenedithiol (BzDT). All of these ligands have thiol (-SH) end groups utilizing the same binding mechanism. However, they vary in length (HT vs. DDT), are either monofunctionalized or bifunctionalized (HT vs. HDT), or have a chemically different backbone at comparable ligand lengths (BzDT vs. HDT).

To achieve insights into ligand dynamics over a range of time scales, we perform QENS measurements on each sample using two different instruments (Figure 1c): (1) the time-of flight spectrometer FOCUS at the Paul Scherrer Institute (PSI), Switzerland, where we can observe processes in the picosecond regime, and (2) the backscattering-spectrometer IN16B at the Institut Laue-Langevin (ILL), France, which can capture slower processes in the nanosecond regime in a narrow energy window. 45

On the basis of the dynamics of hydrogen atoms observed in the QENS measurements, we establish a quantitative model for the motion of thiol-based ligands in NC solids. The model parameters enable us to identify trends in ligand motion as a function of its length, the number of binding groups, and the type of backbone and draw conclusions about how different ligands tether to NC surfaces and arrange in solids.

\section{RESULTS AND DISCUSSION}

We first describe our analysis of the neutron scattering data for the case of a PbS NC solid with HDT ligands and propose a model that describes the observed ligand motion. We then apply the procedure to the other ligands and compare them in the discussion.

Analysis of QENS Data. From our measurement, due to the large incoherent scattering cross-section of hydrogen atoms compared to the scattering cross sections of other elements in our sample $\left(\sigma_{\mathrm{H}, \text { inc }}=80.26\right.$ barn $>\sigma_{\mathrm{Pb} \text {, inc }}=11.12$ barn $){ }^{46}$ we effectively obtain the incoherent structure factor $S^{\text {inc }}$ of hydrogen atoms. On the basis of literature reports on other NC systems, ${ }^{39,40,47}$ we expect a fraction of molecules, $p_{x}$, that move as well as a fraction of molecules, $1-p_{x}$, that are static in 


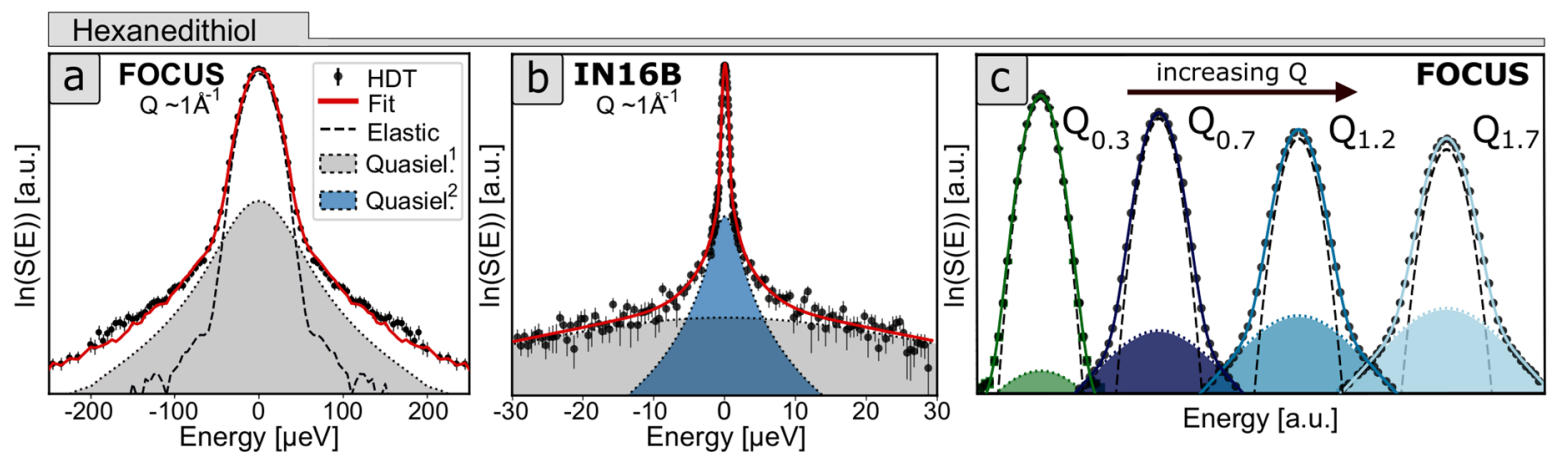

Figure 2. Analysis of quasi-elastic neutron scattering spectra (QENS). (a) QENS spectra of a PbS nanocrystal solid with HDT ligands taken on FOCUS at $300 \mathrm{~K}$ (black dots), with the best fit (solid-red) and fit components: the elastic contribution convoluted with the instrumental resolution function (dashed black line) and the quasi-elastic contribution convoluted with the instrumental resolution function (shaded gray). (b) Same as panel a for the data from IN16B with a second quasi-elastic contribution (shaded blue). (c) QENS spectra for several different $Q$ values, $0.3,0.7,1.2$, and $1.7 \AA^{-1}$ (black dots), that are arbitrarily shifted on the $x$ axis for visualization purposes. Dashed black lines show the elastic contribution. The quasi-elastic contributions (colored shaded regions) all have the same half-width-half-maximum.

the time frame of the measurement and thus only contribute to elastic scattering, described by a delta function $\delta(\omega)$ :

$$
S^{\text {inc }}(Q, \omega)=\mathrm{e}^{-\left\langle u^{2}\right\rangle Q^{2} / 3}\left(\left(1-p_{x}\right) \delta(\omega)+p_{x} S^{\text {act }}(Q, \omega)\right)
$$

The Debye-Waller factor (DWF), $\mathrm{e}^{-\left\langle u^{2}\right\rangle Q^{2} / 3}$, which accounts for the attenuation in the scattering spectra due to harmonic vibrations, depends on the momentum transfer, $Q$ and on the mean square displacement, $\left\langle u^{2}\right\rangle$. The scattering of the active fraction, $S^{\text {act }}$, contains the information about the geometry of the motion through the $Q$-dependent prefactors $A_{0}$ (elastic scattering) and $A_{n}$ (quasi-elastic scattering): ${ }^{48}$

$$
S^{\text {act }}(Q, \omega)=A_{0}(Q) \delta(\omega)+\sum_{n \geq 1} A_{n}(Q) \times L_{n}\left(\omega, \Gamma_{n}\right)
$$

The relaxation time, $\tau=1 / \Gamma$, of the hydrogen motion is inversely proportional to the half-width half-maximum (HWHM), $\Gamma$, of the Lorentzian function describing the quasi-elastic contribution. It indicates the average time the ligand spends in a configuration before occupying a new orientation.

The full expressions for the dynamic structure factor and detailed aspects of the fitting routine can be found in the SI; however, here we describe the key findings from the analysis and how parameters describing the motion can be obtained.

A representative spectrum acquired at FOCUS for HDT at $300 \mathrm{~K}$ and $Q=1.0 \AA^{-1}$ (in the middle of the measured $Q$ range) is plotted in Figure $2 \mathrm{a}$. The spectrum consists of an elastic contribution (black dashed) and a quasi-elastic contribution (gray shaded region), both convoluted with the instrument resolution. The QENS contribution can be fitted with a single Lorentzian. Its HWHM corresponds to a relaxation time of $21.0 \pm 0.5$ ps. In Figure $2 b$, a representative spectrum acquired at IN16B for HDT at $300 \mathrm{~K}$ and $Q=1.0$ $\AA^{-1}$ is plotted. Two Lorentzians are required to fit these spectra, a broad one with the same HWHM as for the spectra recorded at FOCUS (shaded in gray) and a much narrower one (shaded in blue). We attribute the contribution found on both instruments (gray regions) to the same rotational process. The narrower (slower) component can only be resolved at IN16B, and its HWHM corresponds to a relaxation time of $390 \pm 20$ ps for HDT. These results indicate that hydrogen atom motion occurs with two different time constants $(\sim 21 \mathrm{ps}$ and $\sim 390 \mathrm{ps}$ ).

By studying the spectra as a function of $Q$ it is possible to learn about the type of motion the hydrogen atoms undergo. For example, a $Q$-independent HWHM indicates a localized motion of the hydrogen atoms (e.g., stemming from ligand rotation), while a $Q$-dependent HWHM indicates long-range diffusion of hydrogen atoms (e.g., diffusion of ligands on the NC surface or in the sample). ${ }^{48}$ As highlighted in Figure $2 c$, which shows a few representative spectra from FOCUS at different $Q$ values (dotted) with their corresponding fits (solid lines), both the spectra from FOCUS and IN16B show a $Q$ independent HWHM. This tells us that in our PbS NC solid with HDT the ligands stay fixed to their binding site and we can exclude effects of unbound ligands, ${ }^{53}$ which is consistent with the strong binding expected for thiol ligands. ${ }^{55}$ Furthermore, by considering the change in the ratio of the elastic $A_{0}$ to quasi-elastic $A_{n}$ intensity as a function of $Q$ it is possible to quantify the type of localized motion. This ratio is referred to as the elastic incoherent structure factor (EISF): ${ }^{48}$

$$
\operatorname{EISF}(Q)=\frac{A_{0}(Q)}{A_{0}(Q)+\sum_{n \geq 1} A_{n}(Q)}
$$

Model for Ligand Rotation. On the basis of previous QENS studies of surface tethered alkanethiols ${ }^{39,40,47}$ and mathematical models developed for liquid crystals, ${ }^{37,49}$ we propose a model, based on uniaxial rotation and precession, to explain the two types of observed motion, which is visualized in Figure 3a. Other models that we examined, such as isotropic or restricted motion of the ligand in a sphere, do not fit the EISF adequately (SI). Furthermore, the absence of a specific $N$-fold jump rotation (for the case of HDT) indicates that the ligand motion is continuous rather than following a discrete geometrical orientation, which could have stemmed from the ligand itself or its surrounding environment.

The first motion type is the uniaxial rotation of the hydrogen atoms around the axis of the carbon backbone (M1). From the geometrical relaxation (DFT simulation) $)^{50}$ of an HDT molecule in all-trans configuration, we can determine the radius of rotation, i.e., the distance from the molecular axis, for each hydrogen atom in the molecule, $a_{i}$. This radius is used to calculate the resulting uniaxial rotation around the chain backbone. Its elastic contribution is expressed as 


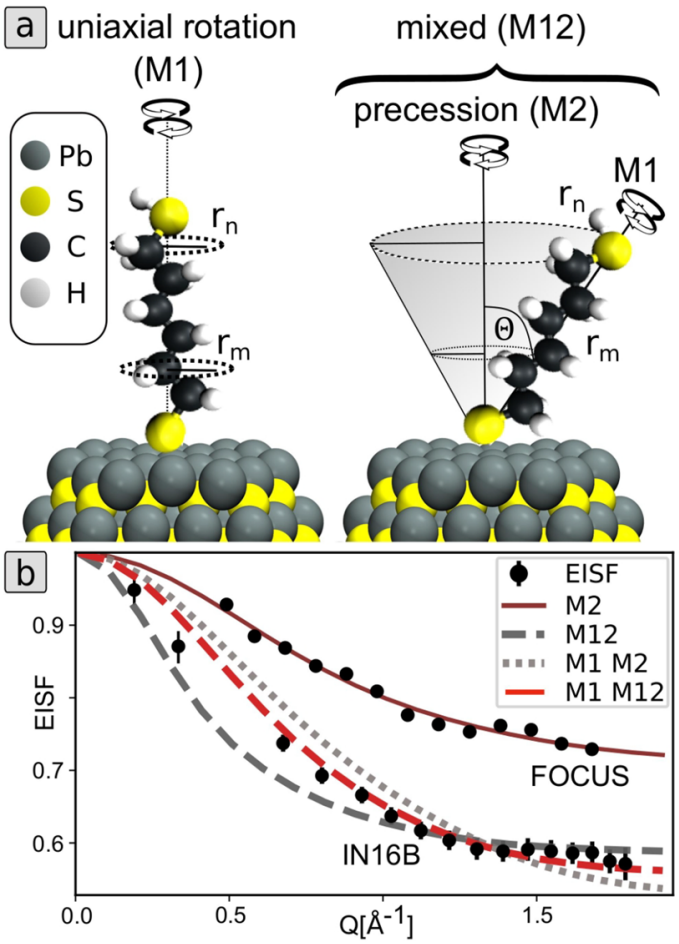

Figure 3. Models for ligand motion. (a) Visualization of the uniaxial rotation (M1), precession (M2), and mixed motion model (M12). In M1, hydrogen atoms rotate around the backbone of the ligand chain at a radius of $r$. In M2, the chain rotates at an opening angle $\theta$, and each hydrogen atom has a rotation radius dependent on its position in the chain. In M12, both motions occur on the same chain. (b) The experimental elastic incoherent scattering factors (EISFs; black dots) for the NC solid with HDT ligands from the two spectrometers. The FOCUS data are best fit with the precession model; M2 (red line) and the IN16B data are best fit with a combination (red dashed line) of some uniaxial rotation M1 and some mixed precession and uniaxial rotation, M12. Less satisfactory fits of the IN16B data are obtained assuming that it can be fully explained by (1) ligands either precessing or rotating, M1 + M2 (short dashed gray line), or (2) by ligands only precessing and rotating, M12 (long dashed gray line).

$$
A_{0}^{M 1}=\frac{1}{N \pi} \sum_{i=1}^{N} \int_{0}^{\pi} j_{0}\left(2 Q a_{i} \sin (\varphi)\right) \mathrm{d} \varphi
$$

where $j_{0}$ is the spherical Bessel function of the first kind and zero order and $N$ is the total number of hydrogen atoms. As the sample is a powder, an isotropic average over the orientational angle $\varphi$ is performed. ${ }^{37}$

The second type of motion is the precession of the alkane chain with an opening angle $\theta$ around a central axis (M2). Similar to eq 3 , we obtain

$$
A_{0}^{\mathrm{M} 2}(\theta)=\frac{1}{N \pi} \sum_{i=1}^{N} \int_{0}^{\pi} j_{0}\left(2 Q r_{i}(\theta) \sin (\varphi)\right) \mathrm{d} \varphi
$$

where the radius, $r_{i}(\theta)$, is the perpendicular distance of a hydrogen atom to the axis of rotation.

In Figure 3b, we show how the individual models or combinations of the two models (M1 and M2) fit to the experimental EISFs. The EISF obtained from the experiment at FOCUS indicates that the fast rotational process is well described by a molecular precession (M2). The backscattering data from IN16B also contains information on a second, slower process. Such a signal could stem (a) from a second independent motion, nonprecessing chains undergo uniaxial rotation ( $\mathrm{M} 1+\mathrm{M} 2)$; (b) from a coupled motion, hydrogen atoms on the precessing chains rotate simultaneously around the molecule axis (M12); or (c) or a mixture of those, namely, hydrogen atoms on precessing chains simultaneously rotate around the molecular axis and additionally a fraction of chains only undergoes uniaxial rotation $(\mathrm{M} 1+\mathrm{M} 12)$. To describe the two localized coupled rotations (M12), the contributions of the two models are convoluted. From the shape of the EISF, we can determine whether the motion is independent, coupled, or a combination of both. In all cases, we allow for part of the molecules not to rotate, referred to as static ligands, which do not rotate even for the longer observation times at IN16B.

We find that the mixed model (case c, M1 + M12) gives the best fit to the IN16B experimental EISF (Figure 3b), where the EISF can be written as

$$
\operatorname{EISF}=p_{1}\left(A_{0}^{\mathrm{M} 1}\right)+p_{12}\left(A_{0}^{\mathrm{M} 1} A_{0}^{\mathrm{M} 2}(\theta)\right)+\left(1-p_{1}-p_{12}\right)
$$

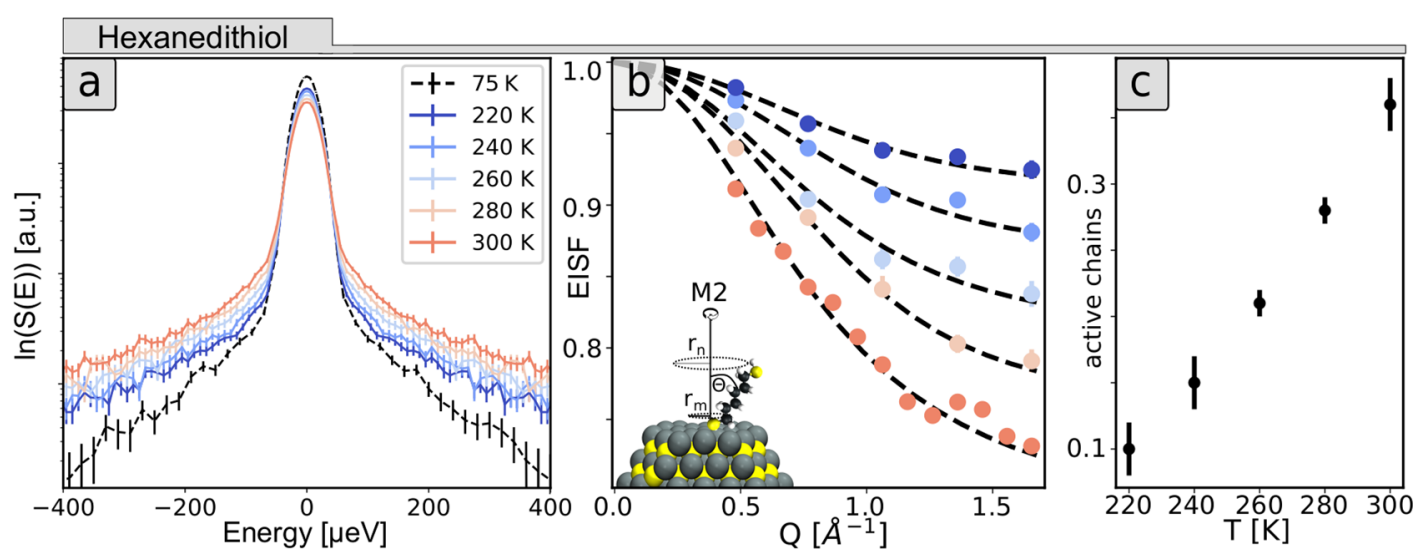

Figure 4. Temperature dependency of ligand motion. (a) Semilogarithmic plot of the scattering spectra obtained at FOCUS for 75K and a temperature series from 220 to $300 \mathrm{~K}$. (b) Q-dependent EISF for different temperatures (colored dots) fit with the precession model (black lines). Fewer data points are available at lower temperatures since a wider Q-binning was chosen due to the lower neutron count at lower temperatures. (c) The fraction of active ligands as a function temperature, obtained by fitting the experimental EISFs in panel $b$ using the M2 precession model. 
Fitting the model to the experimental EISF provides information on the fraction of precessing chains $\left(p_{12}\right)$, fraction of chains performing only uniaxial rotation $\left(p_{1}\right)$, and the fraction of static chains $\left(1-p_{1}-p_{12}\right)$.

In the case of HDT at $300 \mathrm{~K}$, the model indicates that $10-$ $15 \%$ of the ligands are exclusively rotating uniaxially, while $38 \%$ precess and rotate uniaxially simultaneously. The precession occurs at an opening angle of $15^{\circ}$ and with a relaxation time of $21 \mathrm{ps}$, while the relaxation time of the uniaxial rotation is 390 ps. The remaining $\sim 50 \%$ of ligands are static at $300 \mathrm{~K}$ in the time frame of the measurement and do not contribute to quasielastic scattering.

If the ligands have gauche defects, the different motions could hypothetically occur on the same molecule. For example, a ligand could uniaxially rotate between the binding site and the defect and precess at an angle around the central axis after the defect. However, we perform IR spectroscopy and DSC measurements (SI) and find that, as expected from literature reports, ${ }^{22,51}$ the trans configuration dominates in our ligand systems, and only a small gauche defect population exists. Gauche defects become more dominant at higher temperatures but less so in the temperature region of $300 \mathrm{~K}$ and below. ${ }^{22} \mathrm{We}$ therefore conclude that motions do not stem from different regions of the ligand chains but rather from entire ligand chains rotating differently in different environments on the surface.

Activation Energy of Ligand Rotation. Performing QENS measurements at different temperatures allows us to obtain insight into the percentage of ligands rotating and the activation energies of the rotations. Figure $4 a$ shows the temperature dependent scans taken at FOCUS. The onset of quasi-elastic contributions can be observed starting at about $220 \mathrm{~K}$. We see that with increasing temperature, the quasielastic intensity increases, while the elastic component decreases. Thus, we can obtain information on the temperature dependent activation of the precession by evaluating the EISF at each temperature (Figure $4 \mathrm{~b}$ ). We find that the fraction of active chains increases from $\sim 10 \%$ at $220 \mathrm{~K}$ to $38 \%$ at $300 \mathrm{~K}$, shown in Figure 4c, which is similar to reports on comparable systems. ${ }^{39}$ By fitting the temperature dependence of the relaxation time, we obtain the activation energy, $E_{a}$, for the ligand rotation:

$$
\tau=\tau_{0} \mathrm{e}^{E_{\mathrm{a}} / R T}
$$

where $R$ is the universal gas constant and $\tau_{0}$ is the high temperature limit of the relaxation time. For the precession of the HDT chain, we find an activation energy of $6.5 \mathrm{~kJ} / \mathrm{mol}(67$ $\mathrm{meV})$.

To obtain the activation energies of both motions in a more time efficient manner, we measure inelastic-fixed window scans (IFWS) at IN16B. For IFWS, the scattering intensity at a single energy, $\omega_{\text {off }}$, rather than for a full energy spectrum, is recorded. As shown in Figure 5b, two separate peaks are observed, supporting the QENS findings that the ligands undergo two types of rotational motion. The data can be fit well by a linear background and two contributions, each described by

$$
I_{\omega_{\mathrm{off}}}^{\mathrm{IFWS}}=C \frac{\tau(T)}{1+\omega_{\mathrm{off}}^{2} \tau(T)^{2}}
$$

where $C$ is a constant containing all temperature independent contributions. ${ }^{52}$ From this measurement (see simplifying
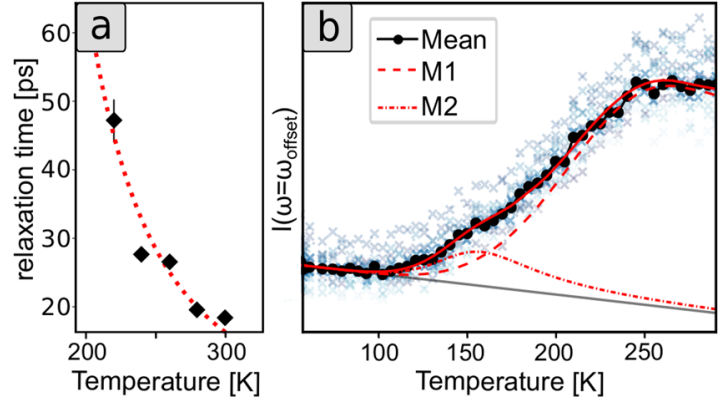

Figure 5. Activation energies of rotational motion. For the PbS NC solid with HDT ligands, we extract the activation energy for rotation in two different ways: (a) First, we determine the activation energy of precession from an Arrhenius-like fit (red dotted line) to the relaxation times of the precession as a function of temperature (black data points). The relaxation times are determined from the HWHMs of the QENS contribution to the scattering factor measured in FOCUS. On the basis of these values, we obtain an activation energy of $6.5 \pm 1.0 \mathrm{~kJ} / \mathrm{mol}$. (b) Second, we estimate the relaxation times for both precession (M2) and uniaxial rotation (M1) by fitting the temperature dependence of the inelastic fixed window scans (IFWS) with a best fit (solid red line) based on two independent quasi-elastic contributions (dashed and dashed-dotted red line) and a linear background (gray). The value obtained for the activation energy of the precession from IFWS is $6.0 \pm 0.5 \mathrm{~kJ} / \mathrm{mol}$ and agrees with the 6.5 $\pm 1.0 \mathrm{~kJ} / \mathrm{mol}$ obtained from the temperature dependent QENS measurements within the error of the analysis.

assumptions in the SI), we extract activation energies for HDT of $\sim 6.0 \pm 0.5 \mathrm{~kJ} / \mathrm{mol}(62 \mathrm{meV})$ for the ligand precession and $\sim 8.0 \pm 0.5 \mathrm{~kJ} / \mathrm{mol}(83 \mathrm{meV})$ for the uniaxial rotation. While IFWS do present some experimental restrictions (SI) compared to the temperature dependent QENS measurement, the value for ligand precession from IFWS is consistent with the value obtained from FOCUS through eq 6.

\section{DISCUSSION: IMPACT OF LIGAND SELECTION}

QENS spectra of the other measured ligands (1-hexanethiol (HT), 1-dodecanethiol (DDT), and 1,4-benzenedithiol (BzDT)) also show Q-independent HWHMs. This confirms the absence of long-range diffusion of ligands for all samples and hence that all ligands strongly tether to the surface. As shown by the good fits to the EISF data in Figure 6a, the model developed for HDT (eqs 3 and 4) is also valid for the other linear alkanes HT and DDT. The model also applies to BzDT with a minor adaption to account for the geometry of the benzene ring. Specifically, to improve the goodness of the fit (SI), for BzDT, we adapt the model exchanging continuous uniaxial rotation for a $180^{\circ}$ jump rotation, which is typical for benzene containing molecules. ${ }^{48,54}$ Precessing BzDT chains, however, do not undergo the jump rotation.

In the following paragraphs, we discuss the trends found as a function of ligand choice by comparing the parameters extracted from fits of the model (Table 1). We note that for $\mathrm{HT}$, DDT, and BzDT, QENS measurements are only performed at $300 \mathrm{~K}$; therefore, activation energies for the motions of $\mathrm{HT}, \mathrm{DDT}$, and BzDT are only calculated from temperature dependent IFWS measurements (SI).

Dithiols Can Cross-Link between NCs, Which Inhibits Ligand Precession. We observe differences between the monothiols (DDT and HT) and dithiols (HDT and BzDT). For monothiols, $p_{1}=0$ in eq 5, meaning that the ligand chains 
a

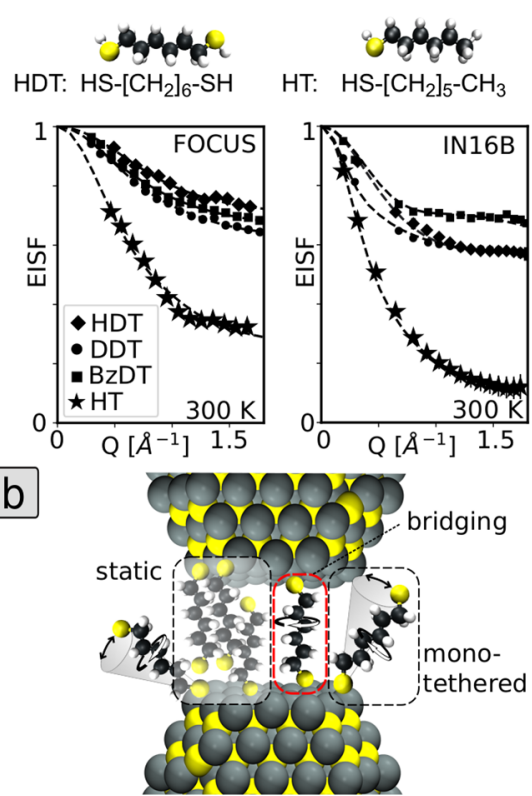

Figure 6. Overview of investigated ligand systems. (a) We investigate (clockwise from upper left): 1,4-benzenedithiol (BzDT), 1-dodecanethiol (DDT), 1-hexanethiol (HT), and 1,6hexanedithiol (HDT). The experimental EISFs extracted from the fits of the data from FOCUS and IN16B spectrometers are shown with fits for models for rotational motion: HDT, diamonds; DDT, circles; BzDT, squares; HT, stars. (b) Visualization of how ligands can connect to $\mathrm{PbS}$ nanocrystal surfaces (e.g., they can bridge or be tethered only to one) and how the motion they undergo (e.g., static, uniaxial rotation, precession, and uniaxial rotation) depends on their local environment.

in these samples only show one motion profile: precession with simultaneous uniaxial rotation on the same chain.

For dithiols, a fraction of $10-20 \%$ of the chains are more restricted in their motion, with hydrogen atoms rotating around the carbon backbone of the chains, without precession. We hypothesize that this confinement in motion is due to the tethering of the ligand at both ends, as illustrated in Figure 6b. The $10-20 \%$ should be treated as a lower bound, as some of the static chains could be tethered at both ends, but their uniaxial rotation is only activated at higher temperatures.

Meanwhile, around $40 \%$ of the dithiol ligands show precession with simultaneous uniaxial rotation on the same chains. We attribute these freely precessing ligands to dithiols that are tethered at only one end due to a difference in their environment compared to the cross-linking ligands. Tethering at only one end of the ligand can happen, for example, if the facet to facet spacing between neighboring NCs is too large for the dithiol to bridge, if neighboring thiol head groups form a disulfide bond, ${ }^{56}$ or if one of the anchoring groups is terminated by a counterion, preventing the cross-linking.

Precession Dynamics. For all ligands, we find relatively short relaxation times of $13-21$ ps and similar opening angles of $15-25^{\circ}$ for the precessing motion, which indicate that these chains generally have space to move quickly and only experience weak chain-to-chain interactions. In comparing HT and HDT, which have the same backbone length, HDT has a smaller opening angle of $15^{\circ}$ (that of HT is $25^{\circ}$ ) and a longer relaxation time of $21 \mathrm{ps}$ (HT has a relaxation time of 15 ps). This indicates that even though these chains do not crosslink, the functional groups at both ends of HDT restrict the precession and the opening angle, possibly via ligands connecting through a disulfide bond.

Some monothiol ligands do not precess within the time frame of our experiments, but we find from the temperature dependent QENS, that with increasing temperature, more of the static chains begin to precess. We hypothesize that at low temperatures, some chains are prevented from rotating either due to interdigitation of the ligand chains with those of neighboring NCs or by ligand-ligand interactions of chains on the same nanocrystal. Indeed, at lower temperatures, linear alkane chains are known to order in bundles on the surface of nanocrystals, with a higher degree of order for longer chains. $^{51,57,58}$

Shorter Chains Are the More Active. Considering the monothiols with different lengths (HT and DDT), we find an activation energy of the precession of $\sim 7.5 \mathrm{~kJ} / \mathrm{mol}(78 \mathrm{meV})$ for $\mathrm{HT}$ and $\sim 9 \mathrm{~kJ} / \mathrm{mol}(93 \mathrm{meV})$ for DDT. At $300 \mathrm{~K}$, the active fraction of DDT is $46 \%$, while the active fraction of HT

Table 1. Insights into Ligand Motion ${ }^{a}$

\begin{tabular}{|c|c|c|c|c|c|c|c|c|}
\hline & \multicolumn{3}{|c|}{ motion distribution } & \multicolumn{2}{|c|}{ uniaxial rotation } & \multicolumn{3}{|c|}{ precession } \\
\hline & $p_{1}[\%]$ & $p_{12}[\%]$ & $\begin{array}{l}p_{\text {static }} \\
{[\%]}\end{array}$ & $\begin{array}{c}\text { relaxation time } \\
{[\mathrm{ps}]}\end{array}$ & $\begin{array}{l}\text { activation energy }[\mathrm{kJ} / \\
\mathrm{mol}]\end{array}$ & $\begin{array}{l}\text { relaxation time } \\
{[\mathrm{ps}]}\end{array}$ & $\begin{array}{l}\text { angle } \\
\text { [deg] }\end{array}$ & $\begin{array}{c}\text { activation energy }[\mathrm{kJ} / \\
\mathrm{mol}]\end{array}$ \\
\hline $\begin{array}{l}\text { hexanedithiol } \\
\text { (HDT) }\end{array}$ & $10-15$ & 38 & $47-52$ & $390 \pm 20$ & $\sim 8.0 \pm 0.5$ & $21 \pm 0.5$ & $15 \pm 4$ & $\begin{array}{l}\quad \sim 6.0 \pm 0.5 \\
\text { FOCUS: } \\
\qquad 6.5 \pm 1.0\end{array}$ \\
\hline $\begin{array}{l}\text { benzenedithiol } \\
\text { (BzDT) }\end{array}$ & $\begin{array}{c}9-18\left(180^{\circ}\right. \\
\text { jump })\end{array}$ & $\begin{array}{c}42 \text { (just } \\
\left.p_{2}\right)\end{array}$ & $38-46$ & $375 \pm 20$ & $\sim 7.5 \pm 0.5$ & $13.5 \pm 0.5$ & $24 \pm 2$ & $\sim 6.5 \pm 0.5$ \\
\hline $\begin{array}{l}\text { dodecanethiol } \\
\text { (DDT) }\end{array}$ & 0 & 46 & 54 & $220 \pm 5$ & $\sim 15.5 \pm 1.0$ & $15 \pm 2$ & $21 \pm 2$ & $\sim 9.0 \pm 0.8$ \\
\hline hexanethiol (HT) & 0 & 90 & 10 & $280 \pm 5$ & $\sim 13.0 \pm 1.0$ & $15 \pm 1$ & $25 \pm 3$ & $\sim 7.5 \pm 0.5$ \\
\hline
\end{tabular}

${ }^{a}$ For the four different ligands studied, information on the fraction of chains rotating at $300 \mathrm{~K}$, their relaxation times, and the activation energy of the motions are given. Specifically, we indicate the percentage of ligands rotating uniaxially $\left(p_{1}\right)$, the percentage of ligands precessing while uniaxially rotating $\left(p_{12}\right)$, and the percentage of ligands not rotating $\left(p_{\text {static }}\right)$ in the timeframe of our observation. For benzedithiol, the uniaxial rotation is replaced by a two-fold jump rotation. We indicate the relaxation times of the rotations and their activation energies as well as the opening angle of the precession. Activation energies are determined from inelastic-fixed window scans (IFWS). For HDT, the activation energy for the precessing motion is additionally extracted from the fitting of the HWHMs of the temperature dependent QENS measurements obtained at FOCUS. 
is $90 \%$. We explain this by the fact that DDT has the longer carbon chain and therefore has stronger interactions with neighboring chains. This possibly results in a higher degree of ordering of the ligands on the nanocrystal surface, which is expected to increase steric hindrance, hindering precession. With increasing temperature, more ligands can break free from their ordered bundles and start precessing. ${ }^{25,39}$ Similarly to DDT, for the dithiols, HDT and BzDT, about half of the chains are still static at $300 \mathrm{~K}$ and only start to precess with increasing temperature, as demonstrated for the case of HDT in Figure 4c.

For all ligands, the activation energy of the uniaxial rotation is higher than that for the precession of the same molecule. For monothiols, all uniaxial rotations occur on chains that are also free to precess, and the precession has an activation energy of 7.5 and $9.0 \mathrm{~kJ} / \mathrm{mol}(78-93 \mathrm{meV})$, while that of uniaxial motion on the same chain is 13.0 and $15.5 \mathrm{~kJ} / \mathrm{mol}(135-160$ $\mathrm{meV})$, respectively. This implies that chains that are only tethered at one end and have the freedom and space to move first begin precessing and then start to rotate around their own axis.

For the dithiols, some of the uniaxial rotations occur on chains which are tethered at both ends, as well as singletethered chains; however, this does not seem to make a difference in the dynamics of the rotation, as we do not observe an additional peak in the IFWS. The uniaxial rotation on precessing and nonprecessing chains appears to be very similar, and we therefore obtain an average relaxation time for the contributions of the uniaxial rotation of both single and double tethered chains.

\section{CONCLUSION}

In summary, we applied QENS to study the motion of different thiol-based ligand species tethered to the surface of PbS NCs, which have been assembled into a solid. We construct a model for ligand motion, and by fitting our model to the experimental data, we are able to draw conclusions about the structure of the ligands on the NC surface, the physical packing of the ligands, their connections, and their dynamics. We obtain a number of insights: (1) Monothiols begin precessing and then rotating while dithiol ligands can either exclusively rotate (indicative of a dithiol that cross-links between nanocrystals) or rotate while precessing (in the case of the ligands that do not bridge), and (2) ligand-ligand interactions, which are defined by the chemical structure of the ligand and the spacing of bonding sites on the NC, will impact the number of active chains (tight packing prohibits motion), the opening angle of the precession, and the speed of the precession and rotation. Such insights can aid, for example, in developing more accurate models for the mechanical and thermal properties of $\mathrm{NC}$ solids, where clear pictures of ligand bonding and packing are critical for describing long-range vibrational motion and phonon scattering. ${ }^{4,18,59}$ More broadly, this study highlights the possibilities of applying QENS to understanding the ligand coverage, bonding, packing, and dynamics on NCs. Neutron spectroscopy can also be extended to study ligand phenomena beyond the scope of this work, such as the dynamics of ligand attachment and detachment, as well as long-range diffusion.

\section{METHODS}

Nanocrystal Synthesis, Ligand Exchange. The $\mathrm{PbS} \mathrm{NC}$ with $3.4 \mathrm{~nm}$ diameter were synthesized using an up-scaled hot-injection approach previously presented in detail. ${ }^{44} \mathrm{~A}$ total of $18 \mathrm{~g}$ of lead oxide were dissolved in $50 \mathrm{~mL}$ of oleic acid and $750 \mathrm{~mL}$ of 1-octadecene inside a three-neck flask, heated to $150{ }^{\circ} \mathrm{C}$, and degassed at the Schlenk line. An $8.4 \mathrm{~mL}$ aliquot of bis(trimethylsilyl)sulfide was mixed with $400 \mathrm{~mL}$ of dried 1-octadecene inside a funnel. The sulfur precursor was injected into the lead solution via vacuum aided injection. During the injection, the solution naturally cooled to 130 ${ }^{\circ} \mathrm{C}$. After $30 \mathrm{~s}$, the heating mantel was removed, and the solution was cooled to $100{ }^{\circ} \mathrm{C}$ over a period of $3 \mathrm{~min}$ and then held at that temperature for another $5 \mathrm{~min}$. Consequently, the solution was then quenched to room temperature with the help of an ice bath. The particles were washed six times using hexane and acetone to remove excess ligands. The ligand exchange and drying of the nanocrystals into a powder was identical to our previous neutron scattering experiments. ${ }^{5,18,60}$

For the ligand exchange, $10-50 \mathrm{~mL}$ of $\mathrm{NC}$ solution $(\sim 90 \mathrm{mg} / \mathrm{mL})$ and $10 \mathrm{~mL}$ of ligand exchange solution $(50 \mathrm{mM}$ in acetonitrile) were mixed and vortexed for $30 \mathrm{~s}$. The NCs were precipitated using methanol and centrifugation ( $9000 \mathrm{rpm}$ for $5 \mathrm{~min}$ ). The supernatant was discarded and the process repeated with a third of the volume of the ligand exchange solution. The particles were then fully dried using the vacuum chamber of the glovebox.

Characterization. We determined a NC diameter of $3.4 \mathrm{~nm}$ via UV-vis spectroscopy (Agilent Cary 5000) and TEM image analysis (JEOL JEM-1400 Plus). To confirm a successful ligand exchange, Fourier-transform infrared (FTIR) spectroscopy (Bruker Vertex 70) was performed on all samples and compared to the as-synthesized particles. Differential scanning calorimetry (TA Instruments DSC 2500) was used to test for glass transitions, melting, and crystallization behavior in the ligand chains adsorbed to the NC surface. Scans were performed at $1 \mathrm{~K} / \mathrm{min}$ from 183 to $323 \mathrm{~K}$. Details of all characterizations can be found in the SI.

Density Functional Theory Calculations of Thiol Ligands and Visualization. The geometry optimization of the ligand molecules to determine the distance of the hydrogen atoms to the molecular axis was done in the $\mathrm{CP} 2 \mathrm{~K}$ program suite utilizing the quickstep module. ${ }^{50}$ Calculations were carried out using a dual basis of localized Gaussians and plane waves, ${ }^{61}$ with a 300 Ry plane-wave cutoff. double-zeta-valence-polarization (DZVP), ${ }^{62}$ GoedeckerTeter-Hutter pseudopotentials ${ }^{63}$ for core electrons, and the Perdew-Burke-Ernzerhof (PBE) exchange correlation functional were used for all calculations. Convergence to $10^{-8}$ in self-consistent field calculations was always enforced. The visualization of the $\mathrm{PbS}$ nanocrystals and ligands was done with the Visual Molecular Dynamics software (VMD). ${ }^{64}$

Neutron Scattering. QENS spectra were acquired as a function of the momentum transfer, $Q\left(0.2-1.8 \AA^{-1}\right)$, and energy transfer (FOCUS, restricted energy range for QENS analysis: $\pm 1 \mathrm{meV}$, IN16B: $\pm 30 \mu \mathrm{eV}$ ) at both $300 \mathrm{~K}$ and at a low temperature (FOCUS: $40 \mathrm{~K}$, IN16B: $10 \mathrm{~K})$. The neutron wavelength was set to $6 \AA$ at FOCUS and $6.27 \AA$ at IN16B. ${ }^{65}$ The dried powder samples were packed in aluminum foil inside an annular aluminum sample holder $\left(d_{\mathrm{o}}=12 \mathrm{~mm}, h=40-50 \mathrm{~mm}\right)$. For the HDT capped NCs, QENS spectra at FOCUS were additionally taken at 220,240, 260, and 280 $\mathrm{K}$. At IN16B, temperature dependent elastic and inelastic fixed window scans, offset by $1.3 \mu \mathrm{eV}$ (FWS and IFWS, respectively), were also performed, which is an efficient method to extract the temperature dependence of the diffusion parameters. Further details on the experimental conditions at both spectrometers and about the analysis of the scattering data can be found in the SI.

\section{ASSOCIATED CONTENT}

\section{(s) Supporting Information}

The Supporting Information is available free of charge at https://pubs.acs.org/doi/10.1021/acsnano.1c09073.

Nanocrystal characterization, neutron scattering measurements, data treatment, alternative models, as well as the results from infrared spectroscopy and calorimetry (PDF) 


\section{AUTHOR INFORMATION}

\section{Corresponding Authors}

Fanni Juranyi - Laboratory for Neutron Scattering and Imaging, Paul Scherrer Institute, CH-5232 Villigen PSI, Switzerland; $\odot$ orcid.org/0000-0002-3223-072X; Email: fanni.juranyi@psi.ch

Vanessa Wood - Department of Information Technology and Electrical Engineering, ETH Zurich, CH-8092 Zurich, Switzerland; $\odot$ orcid.org/0000-0001-6435-0227; Email: vwood@ethz.ch

\section{Authors}

Maximilian Jansen - Department of Information Technology and Electrical Engineering, ETH Zurich, CH-8092 Zurich, Switzerland; @ orcid.org/0000-0003-0610-5439

Olesya Yarema - Department of Information Technology and Electrical Engineering, ETH Zurich, CH-8092 Zurich, Switzerland; ๑ orcid.org/0000-0002-1653-1338

Tilo Seydel - Institut Laue-Langevin (ILL), 38042 Grenoble Cedex 9, France; ๑ orcid.org/0000-0001-9630-1630

Complete contact information is available at:

https://pubs.acs.org/10.1021/acsnano.1c09073

\section{Author Contributions}

The manuscript was written through contributions of all authors. All authors have given approval to the final version of the manuscript.

\section{Notes}

The authors declare no competing financial interest.

\section{ACKNOWLEDGMENTS}

The authors acknowledge support from the Swiss National Science Foundation through Project No. 175889, from the Quantum Sciences and Technology NCCR. This work is based on experiments performed at the Swiss spallation neutron source SINQ Paul Scherrer Institute, Villigen, Switzerland, and at IN16B at the Institut Laue-Langevin, Grenoble, France. We wish to thank both the PSI and ILL for making their facilities available to us.

\section{REFERENCES}

(1) Winslow, S. W.; Smilgies, D. M.; Swan, J. W.; Tisdale, W. A. Reversible Temperature-Induced Structural Transformations in PbS Nanocrystal Superlattices. J. Phys. Chem. C 2020, 124, 13456-13466.

(2) Boles, M. A.; Engel, M.; Talapin, D. V. Self-Assembly of Colloidal Nanocrystals: From Intricate Structures to Functional Materials. Chem. Rev. 2016, 116, 11220-11289.

(3) Talapin, D. V.; Lee, J.-S.; Kovalenko, M. V.; Shevchenko, E. V. Prospects of Colloidal Nanocrystals for Electronic and Optoelectronic Applications. Chem. Rev. 2010, 110, 389-458.

(4) Ong, W.-L.; Rupich, S. M.; Talapin, D. V.; McGaughey, A. J. H.; Malen, J. A. Surface Chemistry Mediates Thermal Transport in Three-Dimensional Nanocrystal Arrays. Nat. Mater. 2013, 12, 410415.

(5) Bozyigit, D.; Yazdani, N.; Yarema, M.; Yarema, O.; Lin, W. M. M.; Volk, S.; Vuttivorakulchai, K.; Luisier, M.; Juranyi, F.; Wood, V. Soft Surfaces of Nanomaterials Enable Strong Phonon Interactions. Nature 2016, 531, 618-622.

(6) Moreels, I.; Justo, Y.; De Geyter, B.; Haustraete, K.; Martins, J. C.; Hens, Z. Size-Tunable, Bright, and Stable PbS Quantum Dots: A Surface Chemistry Study. ACS Nano 2011, 5, 2004-2012.

(7) Mack, T. G.; Jethi, L.; Andrews, M.; Kambhampati, P. Direct Observation of Vibronic Coupling between Excitonic States of CdSe
Nanocrystals and Their Passivating Ligands. J. Phys. Chem. C 2019, 123, 5084-5091.

(8) Zhang, X.; Santra, P. K.; Tian, L.; Johansson, M. B.; Rensmo, H.; Johansson, E. M. J. Highly Efficient Flexible Quantum Dot Solar with Improved Electron Extraction Using $\mathrm{MgZnO}$ Nanocrystals. ACS Nano 2017, 11, 8478-8487.

(9) Wu, W.; Shevchenko, E. V. The Surface Science of Nanoparticles for Catalysis: Electronic and Steric Effects of Organic Ligands. J. Nanopart. Res. 2018, 20, 255.

(10) Kwon, S. G.; Krylova, G.; Sumer, A.; Schwartz, M. M.; Bunel, E. E.; Marshall, C. L.; Chattopadhyay, S.; Lee, B.; Jellinek, J.; Shevchenko, E. V. Capping Ligands as Selectivity Switchers in Hydrogenation Reactions. Nano Lett. 2012, 12, 5382-5388.

(11) Guntern, Y. T.; Okatenko, V.; Pankhurst, J.; Varandili, S. B.; Iyengar, P.; Koolen, C.; Stoian, D.; Vavra, J.; Buonsanti, R. Colloidal Nanocrystals as Electrocatalysts with Tunable Activity and Selectivity. ACS Catal. 2021, 11, 1248-1295.

(12) Volk, S.; Yazdani, N.; Wood, V. Manipulating Electronic Structure from the Bottom-Up: Colloidal Nanocrystal-Based Semiconductors. J. Phys. Chem. Lett. 2020, 11, 9255-9264.

(13) Harris, R. D.; Bettis Homan, S.; Kodaimati, M.; He, C.; Nepomnyashchii, A. B.; Swenson, N. K.; Lian, S.; Calzada, R.; Weiss, E. A. Electronic Processes within Quantum Dot-Molecule Complexes. Chem. Rev. 2016, 116, 12865-12919.

(14) Kestell, J.; Abuflaha, R.; Garvey, M.; Tysoe, W. T. SelfAssembled Oligomeric Structures from 1,4-Benzenedithiol on $\mathrm{Au}(111)$ and the Formation of Conductive Linkers between Gold Nanoparticles. J. Phys. Chem. C 2015, 119, 23042-23051.

(15) Klem, E. J. D.; Shukla, H.; Hinds, S.; MacNeil, D. D.; Levina, L.; Sargent, E. H. Impact of Dithiol Treatment and Air Annealing on the Conductivity, Mobility, and Hole Density in $\mathrm{PbS}$ Colloidal Quantum Dot Solids. Appl. Phys. Lett. 2008, 92, 212105.

(16) Podsiadlo, P.; Krylova, G.; Lee, B.; Critchley, K.; Gosztola, D. J.; Talapin, D. V.; Ashby, P. D.; Shevchenko, E. V. The Role of Order, Nanocrystal Size, and Capping Ligands in the Collective Mechanical Response of Three-Dimensional Nanocrystal Solids. J. Am. Chem. Soc. 2010, 132, 8953-8960.

(17) Jansen, M.; Yazdani, N.; Wood, V. Phonon-Engineered Solids Constructed from Nanocrystals. APL Mater. 2019, 7, 081124.

(18) Yazdani, N.; Jansen, M.; Bozyigit, D.; Lin, W. M. M.; Volk, S.; Yarema, O.; Yarema, M.; Juranyi, F.; Huber, S. D.; Wood, V. Nanocrystal Superlattices as Phonon-Engineered Solids and Acoustic Metamaterials. Nat. Commun. 2019, 10, 4236.

(19) Ong, W.-L.; Majumdar, S.; Malen, J. A.; McGaughey, A. J. H. Coupling of Organic and Inorganic Vibrational States and Their Thermal Transport in Nanocrystal Arrays. J. Phys. Chem. C 2014, 118, $7288-7295$.

(20) Liu, M.; Ma, Y.; Wang, R. Y. Modifying Thermal Transport in Colloidal Nanocrystal Solids with Surface Chemistry. ACS Nano 2015, 9, 12079-12087.

(21) Boles, M. A.; Ling, D.; Hyeon, T.; Talapin, D. V. The Surface Science of Nanocrystals. Nature Materials; Nature Publishing Group, 2016; pp 141-153.

(22) Badia, A.; Cuccia, L.; Demers, L.; Morin, F.; Lennox, R. B. Structure and Dynamics in Alkanethiolate Monolayers Self-Assembled on Gold Nanoparticles: A DSC, FT-IR, and Deuterium NMR Study. J. Am. Chem. Soc. 1997, 119, 2682-2692.

(23) Hostetler, M. J.; Wingate, J. E.; Zhong, C.-J.; Harris, J. E.; Vachet, R. W.; Clark, M. R.; Londono, J. D.; Green, S. J.; Stokes, J. J.; Wignall, G. D.; Glish, G. L.; Porter, M. D.; Evans, N. D.; Murray, R. W. Alkanethiolate Gold Cluster Molecules with Core Diameters from 1.5 to $5.2 \mathrm{Nm}$ : Core and Monolayer Properties as a Function of Core Size. Langmuir 1998, 14, 17-30.

(24) Ang, T. P.; Wee, T. S. A.; Chin, W. S. Three-Dimensional SelfAssembled Monolayer (3D SAM) of n-Alkanethiols on Copper Nanoclusters. J. Phys. Chem. B 2004, 108, 11001-11010.

(25) Pang, Z.; Zhang, J.; Cao, W.; Kong, X.; Peng, X. Partitioning Surface Ligands on Nanocrystals for Maximal Solubility. Nat. Commun. 2019, 10, 1-8. 
(26) Loiudice, A.; Segura Lecina, O.; Bornet, A.; Luther, J. M.; Buonsanti, R. Ligand Locking on Quantum Dot Surfaces via a Mild Reactive Surface Treatment. J. Am. Chem. Soc. 2021, 143, 1341813427.

(27) Weidman, M. C.; Yager, K. G.; Tisdale, W. A. Interparticle Spacing and Structural Ordering in Superlattice $\mathrm{PbS}$ Nanocrystal Solids Undergoing Ligand Exchange. Chem. Mater. 2015, 27, 474482.

(28) Weidman, M. C.; Nguyen, Q.; Smilgies, D.-M.; Tisdale, W. A. Impact of Size Dispersity, Ligand Coverage, and Ligand Length on the Structure of PbS Nanocrystal Superlattices. Chem. Mater. 2018, 30, 807-816.

(29) Choi, J. J.; Bealing, C. R.; Bian, K.; Hughes, K. J.; Zhang, W.; Smilgies, D.-M.; Hennig, R. G.; Engstrom, J. R.; Hanrath, T. Controlling Nanocrystal Superlattice Symmetry and Shape-Anisotropic Interactions through Variable Ligand Surface Coverage. J. Am. Chem. Soc. 2011, 133, 3131-3138.

(30) Lin, W. M. M.; Yarema, M.; Liu, M.; Sargent, E.; Wood, V. Nanocrystal Quantum Dot Devices: How the Lead Sulfide (Pbs) System Teaches Us the Importance of Surfaces. Chimia 2021, 75, 398-413.

(31) Luo, Z.; Marson, D.; Ong, Q. K.; Loiudice, A.; Kohlbrecher, J.; Radulescu, A.; Krause-Heuer, A.; Darwish, T.; Balog, S.; Buonsanti, R.; Svergun, D. I.; Posocco, P.; Stellacci, F. Quantitative 3D Determination of Self-Assembled Structures on Nanoparticles Using Small Angle Neutron Scattering. Nat. Commun. 2018, 9, 1-10.

(32) Lee, B.; Littrell, K.; Sha, Y.; Shevchenko, E. V. Revealing the Effects of the Non-Solvent on the Ligand Shell of Nanoparticles and Their Crystallization. J. Am. Chem. Soc. 2019, 141, 16651-16662.

(33) Weir, M. P.; Toolan, D. T. W.; Kilbride, R. C.; Penfold, N. J. W.; Washington, A. L.; King, S. M.; Xiao, J.; Zhang, Z.; Gray, V.; Dowland, S.; Winkel, J.; Greenham, N. C.; Friend, R. H.; Rao, A.; Ryan, A. J.; Jones, R. A. L. Ligand Shell Structure in Lead SulfideOleic Acid Colloidal Quantum Dots Revealed by Small-Angle Scattering. J. Phys. Chem. Lett. 2019, 10, 4713-4719.

(34) Winslow, S. W.; Shcherbakov-Wu, W.; Liu, Y.; Tisdale, W. A.; Swan, J. W. Characterization of Colloidal Nanocrystal Surface Structure Using Small Angle Neutron Scattering and Efficient Bayesian Parameter Estimation. J. Chem. Phys. 2019, 150, 244702.

(35) Seydel, T.; Koza, M. M.; Matsarskaia, O.; André, A.; Maiti, S.; Weber, M.; Schweins, R.; Prévost, S.; Schreiber, F.; Scheele, M. A Neutron Scattering Perspective on the Structure, Softness and Dynamics of the Ligand Shell of PbS Nanocrystals in Solution. Chem. Sci. 2020, 11, 8875-8884.

(36) Sakai, V. G.; Arbe, A. Quasielastic Neutron Scattering in Soft Matter. Curr. Opin. Colloid Interface Sci. 2009, 14, 381-390.

(37) Dianoux, A. J.; Volino, F.; Hervet, H. Molecular Physics Incoherent Scattering Law for Neutron Quasi-Elastic Scattering in Liquid Crystals Incoherent Scattering Law for Neutron Quasi-Elastic Scattering in Liquid Crystals. Mol. Phys. 1975, 30, 1181-1194.

(38) Roosen-Runge, F.; Hennig, M.; Zhang, F.; Jacobs, R. M. J.; Sztucki, M.; Schober, H.; Seydel, T.; Schreiber, F. Protein SelfDiffusion in Crowded Solutions. Proc. Natl. Acad. Sci. U. S. A. 2011, $108,11815-11820$.

(39) Mukhopadhyay, R.; Mitra, S.; Johnson, M.; Rajeev Kumar, V. R.; Pradeep, T. Evolution of the Alkyl-Chain Dynamics in MonolayerProtected Gold Clusters. Phys. Rev. B: Condens. Matter Mater. Phys. 2007, 75, 75414.

(40) Pradeep, T.; Mitra, S.; Nair, A. S.; Mukhopadhyay, R. Dynamics of Alkyl Chains in Monolayer-Protected $\mathrm{Au}$ and Ag Clusters and Silver Thiolates: A Comprehensive Quasielastic Neutron Scattering Investigation. J. Phys. Chem. B 2004, 108, 7012-7020.

(41) Sandhyarani, N.; Antony, M. P.; Selvam, G. P.; Pradeep, T. Melting of Monolayer Protected Cluster Superlattices. J. Chem. Phys. 2000, 113, 9794-9803.

(42) Moreels, I.; Lambert, K.; Smeets, D.; De Muynck, D.; Nollet, T.; Martins, J. C.; Vanhaecke, F.; Vantomme, A.; Delerue, C.; Allan, G.; Hens, Z. Size-Dependent Optical Properties of Colloidal PbS Quantum Dots. ACS Nano 2009, 3, 3023-3030.
(43) Zherebetskyy, D.; Scheele, M.; Zhang, Y.; Bronstein, N.; Thompson, C.; Britt, D.; Salmeron, M.; Alivisatos, P.; Wang, L.-W. Hydroxylation of the Surface of $\mathrm{PbS}$ Nanocrystals Passivated with Oleic Acid. Science 2014, 344, 1380-1384.

(44) Yarema, M.; Yarema, O.; Lin, W. M. M.; Volk, S.; Yazdani, N.; Bozyigit, D.; Wood, V. Upscaling Colloidal Nanocrystal Hot-Injection Syntheses via Reactor Underpressure. Chem. Mater. 2017, 29, 796803.

(45) Frick, B.; Mamontov, E.; van Eijck, L.; Seydel, T. Recent Backscattering Instrument Developments at the ILL and SNS. Z. Phys. Chem. 2010, 224, 33-60.

(46) Sears, V. F. Neutron Scattering Lengths and Cross Sections. Neutron News 1992, 3, 26-37.

(47) Mukhopadhyay, R.; Mitra, S.; Pradeep, T.; Tsukushi, I.; Ikeda, $S$. Absence of the Rotator Phase and Evolution of Dynamical Motions in Cluster Monolayers. J. Chem. Phys. 2003, 118, 4614-4619.

(48) Bée, M. Quasielastic Neutron Scattering: Principles and Applications in Solid State Chemistry, Biology and Materials Science; CRC Press: Bristol, 1988.

(49) Volino, F.; Dianoux, A. J.; Hervet, H. Neutron Quasi-Elastic Scattering Study of Rotational Motions in the Smectic C, H and VI Phases of Terephtal-Bis-Butyl-Aniline (TBBA). J. Phys. Colloques 1976, 37, C3-55-C3-64.

(50) VandeVondele, J.; Krack, M.; Mohamed, F.; Parrinello, M.; Chassaing, T.; Hutter, J. Quickstep: Fast and Accurate Density Functional Calculations Using a Mixed Gaussian and Plane Waves Approach. Comput. Phys. Commun. 2005, 167, 103-128.

(51) Love, J. C.; Estroff, L. A.; Kriebel, J. K.; Nuzzo, R. G.; Whitesides, G. M. Self-Assembled Monolayers of Thiolates on Metals as a Form of Nanotechnology. Chem. Rev. 2005, 105, 1103-1170.

(52) Frick, B.; Combet, J.; Van Eijck, L. New Possibilities with Inelastic Fixed Window Scans and Linear Motor Doppler Drives on High Resolution Neutron Backscattering Spectrometers. Nucl. Instrum. Methods Phys. Res., Sect. A 2012, 669, 7-13.

(53) Winslow, S. W.; Swan, J. W.; Tisdale, W. A. The Importance of Unbound Ligand in Nanocrystal Superlattice Formation. J. Am. Chem. Soc. 2020, 142 (21), 9675-9685.

(54) Kolokolov, D. I.; Maryasov, A. G.; Ollivier, J.; Freude, D.; Haase, J.; Stepanov, A. G.; Jobic, H. Uncovering the Rotation and Translational Mobility of Benzene Confined in UiO-66 (Zr) MetalOrganic Framework by the 2 H NMR-QENS Experimental Toolbox. J. Phys. Chem. C 2017, 121, 2844-2857.

(55) Elimelech, O.; Aviv, O.; Oded, M.; Banin, U. A Tale of Tails: Thermodynamics of CdSe Nanocrystal Surface Ligand Exchange. Nano Lett. 2020, 20, 6396-6403.

(56) Kutsenko, V. Y.; Lopatina, Y. Y.; Bossard-Giannesini, L.; Marchenko, O. A.; Pluchery, O.; Snegir, S. V. Alkylthiol SelfAssembled Monolayers on $\mathrm{Au}(111)$ with Tailored Tail Groups for Attaching Gold Nanoparticles. Nanotechnology 2017, 28, 235603.

(57) Terrill, R. H.; Postlethwaite, T. A.; Chen, C.-h.; Poon, C.-D.; Terzis, A.; Chen, A.; Hutchison, J. E.; Clark, M. R.; Wignall, G.; Londono, J. D.; Superfine, R.; Falvo, M.; Johnson, C. S.; Samulski, E. T.; Murray, R. W. Monolayers in Three Dimensions: NMR, SAXS, Thermal, and Electron Hopping Studies of Alkanethiol Stabilized Gold Clusters. J. Am. Chem. Soc. 1995, 117, 12537-12548.

(58) Hostetler, M. J.; Stokes, J. J.; Murray, R. W. Infrared Spectroscopy of Three-Dimensional Self-Assembled Monolayers: N -Alkanethiolate Monolayers on Gold Cluster Compounds. Langmuir 1996, 12, 3604-3612.

(59) Wang, Z.; Singaravelu, A. S. S.; Dai, R.; Nian, Q.; Chawla, N.; Wang, R. Y. Ligand Crosslinking Boosts Thermal Transport in Colloidal Nanocrystal Solids. Angew. Chem., Int. Ed. 2020, 59, 95569563.

(60) Yazdani, N.; Nguyen-Thanh, T.; Yarema, M.; Lin, W. M. M.; Gao, R.; Yarema, O.; Bosak, A.; Wood, V. Measuring the Vibrational Density of States of Nanocrystal-Based Thin Films with Inelastic XRay Scattering. J. Phys. Chem. Lett. 2018, 9, 1561-1567. 
(61) Lippert, G.; Hutter, J.; Parrinello, M. A Hybrid Gaussian and Plane Wave Density Functional Scheme. Mol. Phys. 1997, 92, 477487.

(62) VandeVondele, J.; Hutter, J. Gaussian Basis Sets for Accurate Calculations on Molecular Systems in Gas and Condensed Phases. J. Chem. Phys. 2007, 127, 114105 .

(63) Hartwigsen, C.; Goedecker, S.; Hutter, J. Relativistic Separable Dual-Space Gaussian Pseudopotentials from H to Rn. Phys. Rev. B: Condens. Matter Mater. Phys. 1998, 58, 3641-3662.

(64) Humphrey, W.; Dalke, A.; Schulten, K. VMD: Visual Molecular Dynamics. J. Mol. Graphics 1996, 14, 33-38.

(65) Jansen, M.; Juranyi, F.; Seydel, T. Measuring the Force Constant of Semiconductor Nanocrystal Ligands; Institut Laue-Langevin (ILL), 2020. DOI: 10.5291/ILL-DATA.7-05-500

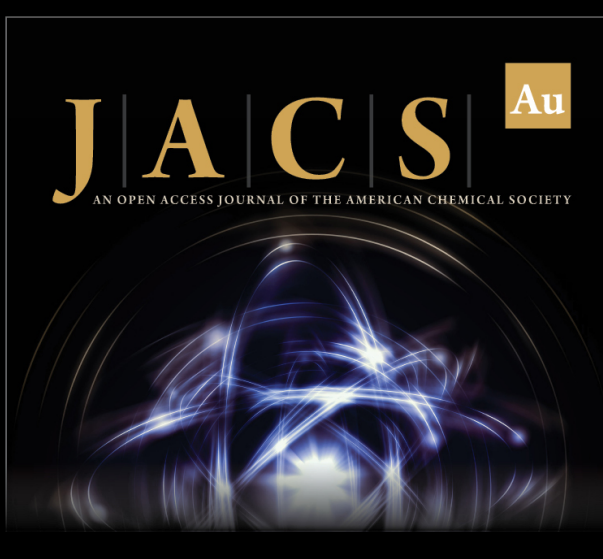

Editor-in-Chief

Prof. Christopher $\mathbf{W}$. Jones

Georgia Institute of Technology, USA

Open for Submissions 